\title{
Experiências Adversas, Trauma, TEPT e Comportamentos de Risco na População e em Veteranos de Guerra
}

\author{
Susana Pedras ${ }^{1}$ \\ Maria da Graça Pereira \\ Escola de Psicologia da Universidade do Minho, Braga, Portugal
}

\begin{abstract}
Resumo
A vivência de experiências adversas na infância, a exposição ao trauma e o quadro clínico de TEPT são fatores de risco para o desenvolvimento de comportamentos de risco (consumo de substâncias, comportamentos sexuais de risco e estilo de vida sedentário). Para além disso, a literatura tem vindo a documentar que existe um fenômeno de transmissão intergeracional de comportamentos de risco dos progenitores para os filhos realçando a importância da intervenção com as vítimas primárias de um trauma devido não só ao risco de envolvimento em comportamentos que prejudicam a saúde mas também devido ao seu poder como modelo parental na transmissão dos comportamentos de risco. Este trabalho apresenta estudos, na área do stress traumático na população geral e em veteranos de guerra tendo também em consideração a investigação sobre o mecanismo intergeracional dos comportamentos de risco da família de origem para a vida adulta.
\end{abstract}

Palavras-chave: Comportamentos de risco, experiências adversas, trauma, TEPT.

\section{Adverse Experiences, Trauma, PTSD and Risk Behaviors in Popula- tion and War Veterans}

\begin{abstract}
Adverse childhood experiences, trauma exposure and PTSD are risk factors for the development of health risk behaviors (substance use, sexual risk behaviors and sedentary life style). In addition, literature has shown that there is an intergenerational transmission of parent's health risk behaviors to the offspring, emphasizing the importance of intervention with primary victims of trauma due to the risk of their involvement in health damaging behaviors and the power of parents, as roles models, in the intergenerational transmission of risk behaviors. This work presents studies in the area of traumatic stress in the general population and in war veterans taking also in consideration the research regarding the intergeneracional transmission of risk behaviors, from the family of origin to adult life.
\end{abstract}

Keywords: Health risk behaviors, adverse experiences, trauma, PTSD.

\section{Experiencias Adversas, Trauma, PTSD y Comportamientos de Ries- go en la Populación en general y en los Veteranos de Guerra}

\section{Resumen}

Las experiencias adversas en la niñez, la exposición al trauma y el trastorno de estrés postraumático son factores de riesgo para el desarrollo de conductas de riesgo, para la salud (consumo de drogas, compor-

Endereço para correspondência: Escola de Psicologia, Departamento de Psicologia Aplicada, Universidade do Minho, Campus de Gualtar, Braga, Portugal 4710-057. E-mail: susanapedras@gmail.com 
tamientos sexuales de riesgo y estilo de vida sedentario). Además, literatura ha demostrado que existe una transmisión intergeneracional de comportamientos de riesgo para la salud de los padres a los hijos, resaltando la importancia de la intervención con las víctimas principales de traumatismo, debido al riesgo de su participación en conductas perjudiciales para la salud y, el poder de los padres como modelos a seguir en la transmisión intergeneracional de comportamientos de riesgo. Este trabajo presenta estudios en el área del estrés traumático en la población en general y en los veteranos de guerra, llevando, en consideración, la investigación sobre el mecanismo intergeneracional de los comportamientos de riesgo de la familia de origen, para la vida adulta.

Palabras clave: Comportamientos de riesgo, experiencias adversas, trauma, PTSD.

Matarazzo (1984, citado por Ogden, 2004) define os comportamentos de saúde tendo em consideração os hábitos prejudiciais para a saúde bem como os comportamentos de protecção da saúde. Os hábitos prejudiciais para a saúde são os comportamentos patogénicos como por exemplo fumar ou beber álcool em excesso e os comportamentos de protecção para a saúde referem-se aos comportamentos imunogénicos como fazer um check-up. Na verdade, um comportamento de saúde é um comportamento cujo objetivo é impedir o aparecimento de uma doença (Kasl \& Cobb, 1966). Já o comportamento de risco pode ser entendido como o envolvimento e participação em atividades que possam comprometer e prejudicar tanto a saúde física como a mental.

Entre os fatores de risco para o envolvimento em comportamentos de risco estão a exposição a acontecimentos traumáticos e as experiências adversas na infância, bem como a consequente sintomatologia psicopatológica, sobretudo o quadro clínico de transtorno de estresse pós-traumático (TEPT), que podem levar ao desenvolvimento e/ou manutenção de comportamentos de risco para a saúde (Rheingold, Acierno, \& Resnick, 2004). Relativamente às experiências adversas na infância, o estudo de Felitti e colaboradores (1998; The Adverse Childhood Experiences Study - ACE Study) avaliou 9.508 adultos de um centro de cuidados de saúde primários, de forma a perceber a relação entre exposição a experiências adversas na infância, doenças na idade adulta e comportamentos de saúde. Os resultados mostraram que as experiências adversas na infância, como o abuso psicológico, o abuso físico, o abuso sexual, o testemu- nhar violência conjugal nos pais, viver com um adulto familiar toxicodependente, viver com um portador de uma doença mental, suicidário ou ex-condenado/presidiário, raramente ocorriam de forma isolada, sendo frequentemente concomitantes. Assim, são relatados vários tipos de abuso que aumentam o risco de adoção de comportamentos de risco para a saúde bem como a presença de doenças crônicas na idade adulta (Green \& Kimerling, 2004). De fato, os comportamentos de risco para a saúde parecem ter um papel mediador na relação entre a exposição ao trauma e doenças na idade adulta e quanto maior a frequência da exposição ao trauma maior o risco de adoção de comportamentos prejudiciais para a saúde (Felitti et al., 1998; Walker et al., 1999). Felitti e colaboradores (1998) avaliaram dez comportamentos de risco para a saúde, tais como o consumo de tabaco, obesidade severa, inatividade física, humor depressivo, tentativas de suicídio, alcoolismo, consumo de drogas parental, entre outros e relacionou-os com o número de experiências adversas vivenciadas na infância. Os autores verificaram que existe uma relação positiva entre a exposição ao trauma na infância e a adoção de comportamentos de risco na idade adulta dado que a vivência de quatro ou mais experiências adversas na infância aumentava o risco quatro a doze vezes de adotar comportamentos de risco para a saúde (ex: alcoolismo, consumo de drogas, relatar saúde pobre, ter cinquenta ou mais parceiros conjugais, risco $1,4 \mathrm{a}$ 1,6 vezes maior de inatividade física e obesidade entre outros). Ainda segundo este estudo, o número de experiências adversas na infância estava relacionado com a presença de doenças na idade adulta, tais como doença cardíaca, cancro, doen- 
ça pulmonar, fraturas ósseas (Felitti et al., 1998). Posteriormente, abordaremos a questão sobre a exposição ao trauma e as experiências adversas na infância estarem associadas a comportamentos de risco e a doenças na idade adulta. A literatura refere que o mecanismo responsável por esta associação, se baseia nos comportamentos de consumo de drogas, alcool, tabaco e comporta- mentos sexuais de risco que podem ser utilizados como mecanismo de coping perante situações de exposição e vivência de situações traumáticas (Felitti et al., 1998). Se estes mecanismos de coping forem percecionados como eficazes, então a sua utilização será crónica podendo, mais tarde, surgir problemas de saúde fisícos resultantes destes comportamentos de risco.

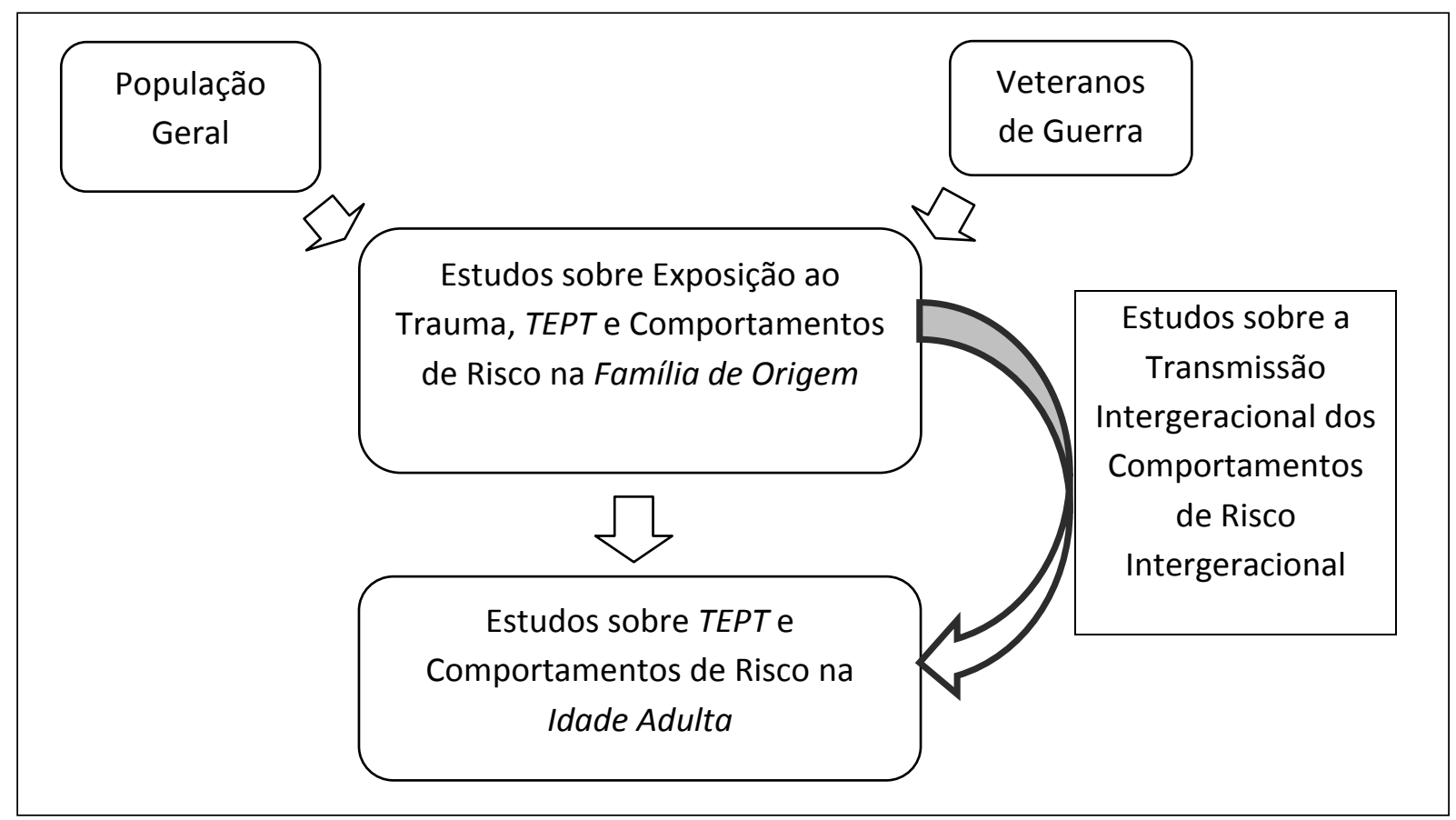

Figura 1. Relação entre exposição ao trauma, TEPT e comportamentos de risco na população geral e veteranos de guerra.

De seguida, abordaremos alguns estudos sobre a relação existente entre a exposição ao trauma, TEPT e comportamentos de risco, na família de origem e a sua adoção na vida adulta, quer na população geral quer em veteranos de guerra ilustrando a transmissão intergeracional dos comportamentos de risco.

\section{Exposição ao Trauma, TEPT e Comportamentos de Risco na População}

Os comportamentos de risco mais referidos na literatura são o consumo de substâncias, os comportamentos sexuais de risco e a adoção de um estilo de vida caracterizado pelo sedentarismo e obesidade (Anda et al., 1999; Dube et al., 2001; Dube, Felitti, Dong, Chapman, et al., 2003; Felitti, 1993; Hillis, Anda, Felitti, \& Marchbanks,
2001; Khoury, Tang, Bradley, Cubells, \& Ressler, 2010; Papastavrou, Farmakas, Karayiannis, \& Kotrotsiou, 2011).

No que diz respeito à exposição ao trauma na infância e o consumo posterior de substâncias, os estudos têm se debruçado sobre o consumo de tabaco, álcool e drogas e a sua relação com a exposição ao trauma e com o TEPT. Em relação ao consumo de tabaco, parece existir uma relação entre a exposição ao trauma, as experiências adversas na infância (abuso emocional, físico e sexual, ter uma mãe agressora, crescer com um elemento da família dependente de substâncias, com uma doença mental entre outros) e o risco de consumir tabaco que é quatro a doze vezes superior comparado com os indivíduos que não relatavam nenhuma experiência adversa (Anda et al., 1999; Weg, 2011). Hernandez (1992) e Walker e colaboradores (1999) encontraram 
uma probabilidade maior de iniciar o consumo de tabaco em mulheres vítimas de maus-tratos na infância e/ou idade adulta (negligência na infância, abuso sexual, abuso emocional).

Relativamente ao álcool, os estudos indicam uma relação significativa entre a exposição ao trauma e o consumo de álcool (Kilpatrick, Acierno, Resnick, Saunder, \& Best, 1997; Walker et al., 1999) e medicamentos analgésicos (Schwartz et al., 2006). Em estudos retrospectivos, o trauma na infância é considerado um fator de risco para o consumo de álcool em homens e mulheres adultos (Dube, Anda, Felitti, Edwards, \& Croft, 2002; Kendler et al., 2000; Kilpatrick \& Resnick, 1993; Kulka et al., 1990; Rheingold et al., 2004; Walker et al., 1999) mas o abuso na idade adulta aumentava o risco de vir a consumir drogas e a desenvolver toxicodependência (Kendler et al., 2000; Kilpatrick et al., 1997). Os adolescentes que foram abusados na infância tinham uma maior probabilidade de vir a consumir e abusar de álcool, conduzir veículos após beber, e consumir álcool antes de terem relações sexuais (Hernandez, Lodico, \& DiClemente, 1993). A literatura é consistente ao indicar que os indivíduos com TEPT têm uma maior probabilidade de consumir álcool do que os que não têm TEPT (Kessler, Sonnega, Bromet, Hughes, \& Nelson, 1995; Kilpatrick \& Resnick, 1993; Rheingold et al., 2004; Sartor et al., 2010).

No que diz respeito ao consumo de drogas, as experiências adversas como os maus-tratos e abuso na infância aumentam o risco de consumo de drogas ilícitas (Dube, Felitti, Dong, Chapman, et al., 2003; Kilpatrick et al., 1997; Walker et al., 1999; Whitmire \& Harlow, 1996). No estudo de Dube, Felitti, Dong, Chapman e colaboradores (2003), cada uma das dez categorias de experiências adversas na infância avaliadas estavam associadas a uma probabilidade duas a quatro vezes maior de consumo de drogas, aos 14 anos, e a um aumento do risco de consumir, na idade adulta. Nos adolescentes que foram sexualmente abusados, a taxa de drogas intravenosas era 14 vezes superior do que a verificada nos jovens não sexualmente abusados (Cunningham, Stiffman, Dore, \& Earls, 1994). O consumo de drogas, tal como o consumo de álcool estava ainda associado a um aumento do risco de vitimização, por representar um risco directo para a saúde que por sua vez, podia aumentar o risco de nova traumatização (Kilpatrick et al., 1997). O consumo de drogas aumentava também o risco de comportamentos que levam a doenças sexualmente transmissíveis, lesões intencionais ou deliberadas, problemas cardíacos, violência interpessoal, incapacidade e crime (Smith, 2000).

Segundo Felitti (2003), numa visão inovadora, a origem dos comportamentos aditivos está predominantemente associada às experiências adversas vivenciadas na infância isto é, a origem dos comportamentos aditivos está predominantemente associada a uma "dependência de experiências de infância" e não a uma "dependência de substâncias". Felitti (2003) realça o fato dos comportamentos de risco serem uma consequência das experiências adversas na infância. De fato, de acordo com Felitti e colaboradores (1998), as experiências adversas estão na base das dificuldades sociais, emocionais e cognitivas, que por sua vez estão na base da adoção de comportamentos de saúde e risco que podem levar à incapacidade e doença e, na pior das circunstâncias, à morte prematura.

Outro comportamento de risco, descrito na literatura,é o comportamento sexual de risco, como ter relações sexuais desprotegidas e ter vários parceiros, dado que aumentam o risco de problemas de saúde e gravidezes indesejadas (Dietz et al., 1999; Hillis et al., 2001; Zierler et al., 1991). Felitti e colaboradores (1998) verificaram que indivíduos expostos a quatro ou mais categorias de experiências adversas na infância tinham um risco quatro a doze vezes maior de relatar cinquenta ou mais parceiros conjugais bem como doenças sexualmente transmissíveis. Hillis e colaboradores (2001) verificaram que a vivência de experiências adversas na infância estava relacionada com o início da vida sexual aos 15 anos, com a percepção de correr riscos de contaminação pelo HIV e ter trinta ou mais parceiros sexuais. Neste estudo, à medida que aumentava a frequência das experiências adversas na infância, aumentava também o risco de adoção destes comportamentos. Num estudo com resultados semelhantes (Walker et al., 
1999), as mulheres com história de maus-tratos na infância tinham mais probabilidade de ter relações sexuais sem conhecer a história sexual do parceiro. Num outro estudo, as vítimas de abuso sexual podem sentir dificuldades em adotar comportamentos de proteção nas suas relações sexuais consentidas e, por isso, ficarem em risco de contaminação pelo HIV (Acierno, Resnick, \& Kilpatrick, 1997). Além disso, os indivíduos vítimas de abuso sexual na infância tinham um risco quatro vezes mais elevado de praticar prostituição e o dobro de probabilidade de ter HIV (Zierler et al., 1991). De igual modo, os rapazes sexualmente abusados tinham três vezes mais probabilidade de ter comportamento homossexual na adolescência e a história de abuso sexual aumentava quatro vezes o risco de não utilizar preservativo e as meninas não levavam os parceiros a usar (Cunningham et al., 1994).

Dietz e colaboradores (1999) verificaram que a primeira gravidez indesejada estava associada ao abuso psicológico, sexual e físico, ao abuso físico da mãe pelo companheiro e à experiência de violação. As mulheres que relatavam quatro ou mais tipos de abuso na infância tinham uma probabilidade 1,5 superior às que não experienciaram qualquer tipo de abuso, de engravidar sem o desejar na idade adulta (Dietz et al., 1999) e a gravidez na adolescência era 2,6 vezes mais prevalente em mulheres que relatavam em particular, a experiência de abuso sexual na infância (Zierler et al., 1991).

Outros comportamentos que podem ser afetados pela exposição ao trauma na infância são a alimentação e o exercício fisico (Pagoto et al., 2012; Williamson, Thompson, Anda, \& Felitti, 2002). Felitti (1993) verificou que numa amostra de adultos obesos, $25 \%$ relatavam história de abuso sexual na infância, comparando com $6 \%$ do grupo controlo e que os adultos com experiências adversas na infância tinham um risco 1,4 a 1,6 mais elevado de obesidade severa e inatividade física (Felitti et al., 1998). De fato, a história de maus-tratos na infância parece aumentar significativamente a probabilidade de não praticar exercício físico regular, de ter um índice de massa corporal (IMC) elevado (Walker et al., 1999) e apresentar obesidade (Pagoto et al., 2012). Outros estudos têm vindo a encontrar uma relação entre a exposição ao trauma (abuso sexual, violência física e sexual/conjugal na idade adulta) e o desenvolvimento de perturbações alimentares como os episódios de binge e a bulimia (Dansky, Brewerton, Kilpatrick, \& O'Neil, 1997). Numa amostra de 75 obesos, Silva e Maia (2008) verificaram que $88 \%$ da amostra relatava a vivência de pelo menos uma experiência adversa na infância e cerca de $47 \%$ relatava cinco ou mais experiências. Relativamente à presença de TEPT, 14 participantes obesos preenchiam os critérios de diagnóstico de TEPT.

\section{Exposição ao Trauma, TEPT e Comportamentos de Risco em Veteranos da Guerra}

Na população de veteranos de guerra, comparando os que apresentavam TEPT versus os que não tinham tal diagnóstico, os primeiros revelavam um aumento da prevalência de abuso de álcool (Kulka et al., 1990; Shalev, Bleich, \& Ursano, 1990; Schnurr \& Spiro, 1999; Stewart, 1996). Segundo Stewart (1996), a exposição ao trauma tem um efeito indirecto nos problemas de consumo de álcool, através do TEPT. Esta relação é cíclica dado que a traumatização pode levar ao desenvolvimento de TEPT que, por sua vez, pode estar na base da iniciação e/ou exacerbação do abuso de álcool (Rheingold et al., 2004).

Em relação ao consumo de tabaco, Schnurr e Spiro (1999) verificaram uma relação positiva entre a experiência em combate e o consumo de tabaco bem como uma relação direta entre os sintomas de TEPT e o consumo. A taxa de consumo de tabaco em veteranos com TEPT $(57 \%$ a $66 \%$ ) era significativamente mais elevada do que nos veteranos sem o diagnóstico de TEPT (34\% a 37\%; Solomon, 1989, citado por Svetlicky, Solomon, Benbenishty, Levi, \& Lubin, 2010; Shalev et al., 1990). De igual forma, o grupo com TEPT fumava um maior número de cigarros por dia (Shalev et al., 1990).

Os estudos indicam que os veteranos com TEPT têm 6 vezes mais probabilidade de consumir drogas do que os veteranos sem TEPT 
(Kessler et al., 1995; Kulka et al., 1990). Os sintomas de evitação e excitabilidade fisiológica e os distúrbios de sono eram os mais frequentemente associados a esse consumo (Dansky, Brady, \& Roberts, 1994). Saladin, Brady, Dansky e Kilpatrick (1995) verificaram que os indivíduos com consumo de álcool e TEPT exibiam mais sintomas de excitabilidade fisiológica do que aqueles com TEPT e dependentes de cocaína. Parece existir uma associação entre experienciar sintomas de TEPT mais severos e uso de drogas como mecanismo de coping para lidar com o stress intenso, embora o consumo de drogas provoque uma exacerbação dos sintomas. McFall, MacKay e Donovan (1992) verificaram que os sintomas de reexperienciação e evitamento do quadro de TEPT estavam mais associados ao consumo de drogas enquanto os sintomas de excitabilidade fisiológica ao consumo de álcool. Mais recentemente, Possemato, Wade, Andersen e Ouimette (2010), num estudo com veteranos da guerra do Iraque e do Afeganistão verificaram que o TEPT, a depressão e a utilização de substâncias estavam associadas a uma maior incidência de doenças e utilização dos serviços de saúde mental, sugerindo que os veteranos podem ter a sua saúde física e mental em risco. Um dado interessante é o fato de alguns estudos não encontrarem uma relação entre a exposição ao trauma na infầncia e um aumento do risco de dependência de substâncias, na idade adulta, mas terem verificado o papel mediador das perturbações de humor nesta relação (Douglas et al., 2010). Num estudo recente (Zen, Whooley, Zhao, \& Cohen, 2011), a depressão explicou a relação existente entre TEPT e consumo de tabaco, em indivíduos com doença cardiovascular, enfatizando o papel da depressão na relação entre TEPT e comportamentos de risco.

Em relação aos veteranos da Guerra Colonial Portuguesa, a maioria dos veteranos apresentavam 2 a 3 comportamentos de risco para a saúde tais como o consumo de álcool, tabaco, gorduras e sedentarismo (Maia, McIntyre, Pereira, \& Fernandes, 2006). Neste estudo, $81 \%$ dos veteranos relatavam consumir álcool, $23 \%$ tabaco e $88 \%$ relatavam consumir cafeína. Segundo os veteranos, o consumo destas substâncias tinha como objetivo diminuir o desconforto e o mal-estar físico e psicológico.

Howard (2007), num estudo de caso, tentou ilustrar de que forma o comportamento sexual compulsivo pode ser um mecanismo de coping para lidar com as memórias intrusivas. Segundo o autor, o comportamento sexual e a visualização de pornografia são consideradas formas de auto-medicação, tal como o consumo de álcool nos alcoolistas.

Tal como referido para a população em geral, a exposição ao trauma relaciona-se com os valores do IMC (Schnurr, Spiro, \& Paris, 2000). Num estudo com 221 veteranos com TEPT verificou-se que cerca de $83 \%$ tinham sobrepeso ou obesidade tendo em conta o IMC encontrado na população de veteranos e na população geral (Vieweg et al., 2006) sendo maior a probabilidade de encontrar obesidade em mulheres veteranas com TEPT do que sem TEPT (Dobie et al., 2004).

O TEPT é um preditor do envolvimento em comportamentos de risco nos veteranos de guerra e, quanto mais severo o quadro de TEPT, maior a tendência para o envolvimento em comportamentos de risco independentemente do grau de exposição a combate (Hartl, Rosen, Drescher, Lee, \& Gusman, 2005; Svetlicky et al., 2010). Contudo, também a história prévia de exposição ao trauma na infância parece ser um fator de risco para o desenvolvimento de TEPT e adoção de comportamentos de risco. De fato, os veteranos que relatavam experiências adversas na infância apresentavam um risco maior de consumir álcool, tabaco e apresentarem comportamentos autodestrutivos (Iversen et al., 2007). Por sua vez, LeardMann, Smith, e Ryan (2010) também verificaram que os veteranos que relatavam múltiplos tipos de abuso na infância apresentavam maior risco de desenvolver TEPT após o serviço militar. Num outro estudo, 53\% dos soldados Iraquianos entrevistados relatavam a vivência de pelo menos uma experiência adversa na infância e $29 \%$ referiam a exposição a duas ou mais experiências adversas, sendo estes últimos os que apresentavam uma taxa mais elevada de TEPT (Cabrera, Hoge, Bliese, Castro, \& Messer, 2007). 


\section{Transmissão Intergeracional dos Comportamentos de Risco}

Vários estudos debruçaram-se sobre o fenômeno da transmissão intergeracional dos comportamentos de risco de pais para filhos, partindo do pressuposto base que o presenciar a adoção de determinados comportamentos de risco para a saúde por parte dos pais, aumenta o risco, nos filhos, de adoção dos mesmos comportamentos na idade adulta. Exemplos desta última linha de investigação são os estudos com filhos de pais alcoolistas. Neste sentido, a investigação indica que os filhos de pais alcoolistas correm um risco mais elevado de vivenciar outras experiências adversas, tal como a negligência e o abuso, o testemunhar violência doméstica e o estar exposto ao consumo abusivo de drogas, doenças mentais, suicídio e comportamento criminal (Dube et al., 2001). Por outro lado, crescer com um pai alcoolista constitui também um risco elevado para o desenvolvimento do alcoolismo na idade adulta, psicopatologia e outros problemas médicos e sociais (Anda et al., 2002; Dube et al., 2001; Merikangas, Dierker, \& Szatmari, 1998). O estudo de Anda e colaboradores (2002) vem ao encontro destes resultados, uma vez que na sua amostra, crescer com um pai alcoolista aumentava significativamente o risco de vivenciar outras experiências adversas na infância, o consumo de drogas nos adolescentes rapazes. Por sua vez, outro estudo revelou um aumento dos comportamentos desviantes nas meninas que cresceram num ambiente familiar com um pai alcoolista (Ritter, Stewart, Bernet, Coe, \& Brown, 2002). Na verdade, a maior parte dos adultos que apresentavam consumo de álcool, relatava história de alcoolismo parental (Anda et al., 2002). Dube e colaboradores (2002) verificaram que os indivíduos com história de adversidade na infância possuíam o risco de consumir álcool de forma abusiva, relatar história de alcoolismo e casar com um alcoolista, sendo este risco duas a quatro vezes superior na presença de várias experiências adversas na infância. Outros autores referem que as crianças que cresceram num ambiente com consumo de substâncias e consumo regular de tabaco correm um risco maior de vir a desenvolver uma dependência de álcool, co- caína e/ou opióides. O consumo de substâncias, na casa dos pais, duplica o risco de desenvolver uma dependência de substâncias e o consumo de tabaco aumenta em 2/3 o risco de fumar na idade adulta (Douglas et al., 2010; Fergusson, Boden, $\&$ Horwood, 2008).

No caso dos filhos de veteranos praticamente não existem estudos sobre o estilo de vida realizados nesta população. Westerink e Giarratano (1999) estudaram o estilo de vida em filhos de veteranos de guerra com TEPT maiores de 15 anos. Contudo, não verificaram diferenças significativas ao nível do estilo de vida entre os filhos de veteranos e o grupo de controlo, excepto nos hábitos tabágicos e na prática de exercício físico. Em Portugal, numa amostra de 80 filhos adultos de veteranos da Guerra Colonial, cerca de 74\% da amostra relatava a vivência de pelo menos uma experiência familiar negativa na infância (presenciar estados de alcoolismo, assistir a tentativas de suicídio de pai/mãe, receber insultos ou humilhações, ser ameaçado com uma arma de fogo, faca ou outro objeto, entre outras; Pedras, 2009). Relativamente ao estilo de vida, $73 \%$ da amostra estudada adotava bons comportamentos de saúde, principalmente as mulheres (Pedras \& Pereira, 2010) dado que estas apresentam uma orientação para a saúde mais positiva do que os homens, e adotam com mais frequência comportamentos de saúde (Brubaker \& Amherst, 2007). Contudo, o nível de perturbação emocional era elevado nesta população, independentemente do género (Pedras, 2009).

\section{Conclusão}

Se por um lado, a vivência de experiências adversas na infância e o TEPT podem despoletar a adoção de comportamentos de risco, por outro, viver com progenitores com hábitos de estilos de vida pouco saudáveis, pode dar lugar a uma repetição dos comportamentos de risco. Assim, parece existir um processo intergeracional de transmissão de padrões de comportamentos de risco dos progenitores para os filhos (Anda et al., 2002). Neste sentido, a comunidade científica e os profissionais de saúde devem cometer todos os esforços para a detecção precoce das experiências adversas na infância de modo a prevenir 
os comportamentos patogénicos e a doença física na idade adulta. Sendo os postos de saúde, o local onde as pessoas habitualmente recorrem quando sentem algum problema físico ou psicológico, é fundamental que os profissionais de saúde estejam sensibilizados para este fato. Assim, durante a entrevista clínica, é necessário ter em atenção a exposição a situações adversas na infância, a exposição a hábitos e comportamentos de risco parentais, bem como a presença de TEPT e comportamentos de risco atuais. De fato, a natureza próxima e continuada da relação do clinico com o utente, facilita a identificação dos hábitos que caracterizam o seu estilo de vida ao nível do consumo tabaco, álcool, drogas, comportamentos sexuais de risco, atividade física, hábitos alimentares e comportamentos de risco. O objetivo é assim, atenuar os efeitos das experiências adversas e da exposição ao trauma na vida adulta e cessar os comportamentos que prejudicam a saúde. Para além disso, é importante ainda que seja realizado um reencaminhamento do paciente para os serviços de psicologia e psiquiatria para que possam ser trabalhadas as questões relacionadas com o quadro traumático. Por fim, gostaríamos de enfatizar que nos indivíduos onde os comportamentos de risco funcionam como respostas de coping (ainda que disfuncionais), não basta cessar estes comportamentos é necessário também encontrar novos mecanismos de coping mais adaptativos. Este tipo de trabalho é essencial para interromper o ciclo de comportamentos de risco relacionados com o trauma e o risco de nova traumatização.

Para, além disso, a adversidade na infância e, sobretudo os efeitos cumulativos de múltiplos tipos de abusos e a presença de TEPT exercem um grande impacto sobre a saúde física. Os estudos indicam que estes indivíduos possuem um risco aumentado de desenvolver doença cardíaca isquémica, doença oncológica, doença pulmonar, diabetes, fraturas ósseas e doenças decorrentes de respostas inflamatórias crônicas como as doenças autoimunes (doença do cólon irritável, diabetes tipo 1, psoríase, esclerose múltipla, lúpus, artrite reumatóide; Brown et al., 2010; Dube, Felitti, Dong, Giles, \& Anda, 2003).
A importância do acompanhamento psicológico reside acima de tudo no fato dos estados emocionais positivos promoverem percepções, crenças saudáveis, bem-estar físico e motivarem a adoção de comportamentos de saúde (Salovey, Rothman, Detweiler, \& Wayne, 2000). Dentre as várias formas de intervenção no trauma salienta-se a intervenção cognitiva-comportamental considerada uma intervenção de primeira linha no TEPT (Foa, Keane, \& Friedman, 2009; Keane, Marshall, \& Taft, 2006) bem como a intervenção familiar sistémica particularmente num processo que inclui uma fase disjunta e uma fase conjunta (Pereira, 2003; Rosenheck \& Thompson, 1986). Ao nível comportamental, a técnica da exposição tem-se revelado eficaz no tratamento dos sintomas de TEPTsobretudo nos sintomas fisiológicos (apontados como uma das razões para a adoção de comportamentos de risco; Foa et al., 2009).

Por fim, a intervenção numa perspectiva preventiva, e não apenas remediativa, assume um papel preponderante. De fato, a promoção da saúde revela-se um campo de ação vasto. Pedras e Pereira (2011) criaram um programa de promoção da saúde para mulheres de veteranos de guerra com TEPT no sentido de minimizar o impacto do TEPT e aumentar os comportamentos de saúde. A promoção da saúde pode focar-se, em termos de alvos, nos pais, filhos, professores, profissionais de saúde, parceiros e qualquer agente envolvido no processo de desenvolvimento de uma criança. Neste sentido, são necessários programas de promoção da saúde, nos veteranos e nas vítimas de trauma em geral no sentido de minimizar a transmissão intergeracional do trauma e os comportamentos de risco.

Detectar a vivência de experiências adversas na infância, intervir no TEPT precocemente e apoiar as vítimas de trauma são formas de evitar a adoção e futuro desenvolvimento de comportamentos de risco. Assim, esperamos que este artigo possa chamar a atenção para a problemática da exposição ao trauma e do quadro clínico de TEPT, no aumento do risco de adoção de comportamentos que colocam em risco a saúde familiar. 


\section{Referências}

Acierno, R., Resnick, H. S., \& Kilpatrick, D. G. (1997). Health impact of interpersonal violence. Section I: Prevalence rates, case identification, and risk factors for sexual assault, physical assault, and domestic violence in men and women. Behavioral Medicine, 23(2), 53-64.

Anda, R. F., Croft, J. B., Felitti, V. J., Nordenberg, D., Giles, W. H., Williamson, D. F., ...Giovino, G. A. (1999). Adverse childhood experiences and smoking. Journal of American Medical Association, 282(17), 1652-1658.

Anda, R. F., Whitfield, C. L., Felitti, V. J., Chapman, D., Edwards, V. J., Dube, S. R., ...Williamson, D. F. (2002). Alcohol-impaired parents and adverse childhood experiences: The risk of depression and alcoholism during adulthood. Psychiatric Services, 53(8), 1001-1009.

Brown, D. W., Anda, R. F., Felitti, V. J., Edwards, V. J., Malarcher, A. M., Croft, J. B., ...Giles, W. H. (2010). Adverse childhood experiences are associated with the risk of lung cancer: A prospective cohort study. BMC Public Health, 10(20), 1-12.

Brubaker, C., \& Amherst, U. (2007). Gender-role orientation, family structure and health behavior: A focus on physical activity. Family Structure and Health Behavior. Retrieved from http://www.allacademic.com $/ /$ meta/p mla apa research citation/1/8/3/3/7/pages183373/p183373-15.php

Cabrera, O. A., Hoge, C. W., Bliese, P. D., Castro, C. A., \& Messer, S. C. (2007). Childhood adversity and combat as predictors of depression and post-traumatic stress in deployed troops. American Journal of Preventive Medicine, 33(2), 77-82.

Cunningham, R. M., Stiffman, A. R., Dore, P., \& Earls, F. (1994). The association of physical and sexual abuse with HIV risk behaviours in adolescence and young adulthood: Implications for public health. Child Abuse and Neglect, 18, 233-245.

Dansky, B. S., Brady, K. T., \& Roberts, J. T. (1994). Posttraumatic stress disorder and substance abuse: Empirical findings and clinical issues. Substance Abuse, 15, 247-257.

Dansky, B. S., Brewerton, T. D., Kilpatrick, D. G., \& O'Neil, P. M. (1997). The national women's study: Victimization and posttraumatic stress disorder to bulimia nervosa. International Journal of Eating Disorders, 21, 213-228.
Dietz, P. M., Spitz, A. M., Anda, R. F., Williamsom, D. F., McMahon, P. M., Santelli, J. S., ...Kendrick, J. S. (1999). Unintended pregnancy among adult women exposed to abuse or household dysfunction during their childhood. Journal of American Medical Association, 282, 1359-1364.

Dobie, D. J., Kivlahan, D. R., Maynard, C., Bush, K. R., Davis, T. M., \& Bradley, K. A. (2004). Posttraumatic stress disorder in female veterans: Association with self-reported health problems and functional impairment. Archives of Internal Medicine, 164, 394-400.

Douglas, K. R., Chan, G., Gelernter, J., Arias, A. J., Anton, R. F., Weiss, R. D., ...Kranzler, H. R. (2010). Adverse childhood events as risk factors for substance dependence: Partial mediation by mood and anxiety disorders. Addictive Behavior, 35(1), 7-13.

Dube, S. R., Anda, R. F., Felitti, V. J., Croft, J. B., Edwards, V. J., \& Giles, W. H. (2001). Growing up with parental alcohol abuse: Exposure to childhood abuse, neglect and household dysfunction. Child Abuse and Neglect, 25, 1627-1640.

Dube, S. R., Anda, R. F., Felitti, V. J., Edwards, V. J., \& Croft, J. B. (2002). Adverse childhood experiences and personal alcohol abuse as an adult. Addictive Behaviors, 27, 713-725.

Dube, S. R., Felitti, V. F., Dong, M., Chapman, D. P., Giles, W. H., \& Anda, R. F. (2003). Childhood abuse, neglect and household dysfunction and the risk of illicit drug use: The Adverse Childhood Experiences Study. Pediatrics, 111(3), 564-572.

Dube, S. R., Felitti, V. J., Dong, M., Giles, W. H., \& Anda, R. F. (2003). The impact of adverse childhood experiences on health problems: Evidence from four birth cohorts dating back to 1990 . Preventive Medicine, 37, 268-277.

Felitti, V. J. (1993). Childhood sexual abuse, depression and family dysfunction in adult obese patients: A case control study. Southern Medical Journal, 86, 732-736.

Felitti, V. J. (2003). [The origins of addiction: Evidence from the adverse childhood experiences study]. Prax Kinderpsychol Kinder Psychiater, 52(8), 547-559.

Felitti, V. J., Anda, R. F., Nordenberg, D., Williamson, D. F., Spitz, A. M., Edwards, V., ...Marks, J. S. (1998). Relationship of childhood abuse and household dysfunction to many of the lead- 
ing causes of death in adults. The Childhood Experiences (ACE) Study. American Journal of Preventive Medicine, 14(4), 245-258.

Fergusson, D. M., Boden, J. M., \& Horwood, L. J. (2008). The developmental antecedents of illicit drug use: Evidence from a 25-year longitudinal study. Drug and Alcohol Dependence, 96(1-2), 165-177.

Foa, E. B., Keane, T. M., \& Friedman, M. J. (2009). Effective treatments for PTSD: Practice guidelines from the International Society for Traumatic Stress Studies ( $2^{\text {nd }}$ ed.). New York: The Guilford Press.

Green, B. L., \& Kimerling, R. (2004). Trauma, posttraumatic stress disorder and health status. In P. P. Schnurr \& B. L. Green (Eds.), Trauma and health: Physical health consequences of exposure to extreme stress (pp. 13-42). Washington, DC: American Psychological Association.

Hartl, T. L., Rosen, C., Drescher, K., Lee, T. T., \& Gusman, F. (2005). Predicting high-risk behaviors in veterans with posttraumatic stress disorder. Journal of Nervous and Mental Disease, 193, 464-472.

Hernandez, J. T. (1992). Substance abuse among sexually abused adolescents and their families. Journal of Adolescent Health, 13(8), 658-662.

Hernandez, J. T., Lodico, M., \& DiClemente, R. J. (1993). The effects of child abuse and race on risk-taking in male adolescents. Journal of $\mathrm{Na}$ tional Medical Association, 85(8), 593-597.

Hillis, S. D., Anda, R. F., Felitti, V. J., \& Marchbanks, P. A. (2001). Adverse childhood experiences and sexual risk behaviours in women: A retrospective cohort study. Family Planning Perspectives, 33(5), 206-211.

Howard, M. D. (2007). Escaping the pain: Examining the use of sexually compulsive behaviour to avoid the traumatic memories of combat. Sexual Addiction \& Compulsivity, 14, 77-94.

Iversen, A. C., Fear, N. T., Simonoff, E., Hull, L., Horn, O., Greenberg, N., ...Wessely, S. (2007). Influence of childhood adversity on health among male UK military personnel. British Journal of Psychiatry, 191, 506-511.

Kasl, S. V., \& Cobb, S. (1966). Health behavior, illness behavior and sick role behavior: 11 sick role behavior. Archives of Environmental Health, 12, $531-541$
Keane, T., Marshall, A., \& Taft, C. (2006). Posttraumatic stress disorder: Etiology, epidemiology and treatment outcome. Annual Review of Psychology, 2, 161-197.

Kendler, K. S., Bulik, C. M., Silberg, J., Hetterma, J. M., Myers, J., \& Prescott, C. A. (2000). Childhood sexual abuse and adult psychiatric and substance use disorders in women. Archives of General Psychiatry, 57, 953-959.

Kessler, R. C., Sonnega, A., Bromet, E., Hughes, M., \& Nelson, C. B. (1995). Posttraumatic stress disorder in the national comorbidity survey. $A r-$ chives of General Psychiatry, 52(12), 1048-1060.

Khoury, L., Tang, Y., Bradley, B., Cubells, J., \& Ressler, K. (2010). Substance use, childhood traumatic experience, and posttraumatic stress disorder in an urban civilian population. Depression and Anxiety, 27(12), 1077-1086.

Kilpatrick, D. G., Acierno, R., Resnick, H., Saunders, B. E., \& Best, C. (1997). A two-year longitudinal analysis of the relationships among violent assault and substance use in women. Journal of Consulting and Clinical Psychotherapy, 68, 19-30.

Kilpatrick, D. G., \& Resnick, H. S. (1993). Posttraumatic stress disorder associated with exposure to criminal victimization in clinical and community population. In J. R. T. Davidson \& E. B. Foa (Eds.), Posttraumatic stress disorder: DSMIV and beyond (pp. 113-143). Washington, DC: American Psychiatric Press.

Kulka, R. A., Schlenger, W. E., Fairbank, J. A., Hough, R. L., Jordan, B. K., Marmar, C. R., ...Cranston, A. (1990). Trauma and the Vietnam War generation. New York: Brunner Mazel.

LeardMann, C. A., Smith, B., \& Ryan, M. (2010). Do adverse childhood experiences increase the risk of post deployment posttraumatic stress disorder in US Marines? BMC Public Health, 10(437), 1-8

Maia, A., McIntyre, T., Pereira, M. G., \& Fernandes, E. (2006). Por baixo das pústulas da Guerra: Reflexões sobre um estudo com ex-combatentes da Guerra Colonial. In M. Gama, A Guerra Colonial (1961-1974) (pp. 11-28). Braga, Portugal: Centro de Estudos Lusíadas, Universidade do Minho.

McFall, M. E., MacKay, P. W., \& Donovan, D. M. (1992). Combat related posttraumatic stress disorder and severity of substance abuse in Vietnam veterans. Journal of Studies on Alcohol, 53, 357-363. 
Merikangas, K. R., Dierker, L. C., \& Szatmari, P. (1998). Psychopathology among offspring's of parents with substance abuse and/or anxiety disorders: A high-risk study. Journal of Child Psychology and Psychiatry and Allied Disciplines, 39, 711-720.

Ogden, J. (2004). Psicologia da Saúde. Lisboa, Portugal: Climepsi.

Pagoto, S. L., Schneider, K. L., Bodenlos, J. S., Appelhans, B. M., Whited, M. C., Ma, Y., ...Lemon, S. C. (2012). Association of post-traumatic stress disorder and obesity in a nationally representative sample. Obesity, 20(1), 200-205.

Papastavrou, E., Farmakas, A., Karayiannis, G., \& Kotrotsiou, E. (2011). Comorbidity of post-traumatic stress disorders and substance use disorder. Health Science Journal, 5(2), 107-117.

Pedras, S. (2009). Variáveis de saúde, familiares e de psicopatologia em filhos de veteranos da Guerra Colonial Portuguesa (Dissertação de mestrado em Psicologia da Saúde, Escola de Psicologia, Universidade do Minho, Braga, Portugal).

Pedras, S., \& Pereira, M. G. (2010). O papel da adaptabilidade familiar na adoção de comportamentos de saúde em filhos de veteranos de guerra com sintomatologia traumática. In C. Nogueira et al. (Eds.), Actas do VII Simpósio Nacional de Investigação em Psicologia (pp. 1373-1387). Braga, Portugal: Universidade do Minho.

Pedras, S., \& Pereira, M. G. (2011). Promoção da saúde psicossocial em mulheres de veteranos de guerra com PTSD. In M. G. Pereira (Coord.), Promoção da saúde psicossocial na doença: Aspectos teóricos e intervenção (pp. 219-292). Viseu, Portugal: Psicossoma.

Pereira, M. G. (2003). Terapia familiar. In M. G. Pereira \& J. Monteiro-Ferreira (Coords.), Stress traumático: Aspectos teóricos e intervenção (pp. 187-295). Lisboa, Portugal: Climepsi.

Possemato, K., Wade, M., Andersen, J., \& Ouimette, P. (2010). The impact of PTSD, depression, and substance use disorders on disease burden and health care utilization among OEF/OIF veterans. Psychological Trauma: Theory, Research, Practice, and Policy, 2(3), 218-223.

Rheingold, A. A., Acierno, R., \& Resnick, H. S. (2004). Trauma, posttraumatic stress disorder and health risk behaviours. In P. P. Schnurr \& B. L. Green (Eds.), Trauma and health: Physi- cal health consequences of exposure to extreme stress (pp. 217-243). Washington, DC: American Psychological Association.

Ritter, J., Stewart, M., Bernet, C., Coe, M., \& Brown, S. A. (2002). Effects of childhood exposure to familial alcoholism and family violence on adolescent substance use, conduct problems and self-esteem. Journal of Traumatic Stress, 15(2), 113-122.

Rosenheck, R., \& Thomson, J. (1986). Detoxification of Vietnam War trauma: A combined family-individual approach. Family Process, 25, 559-570.

Saladin, M. E., Brady, K. T., Dansky, B. S., \& Kilpatrick, D. G. (1995). Understanding comorbidity between PTSD and substance use disorders: Two preliminary investigations. Addictive Behaviors, 20(5), 643-655.

Salovey, P., Rothman, A. J., Detweiler, J. B., \& Steward, W. T. (2000). Emotional states and physical health. American Psychologist, 55(1), 110-121.

Sartor, C. E., Mccutcheon, V. V., Pommer, N. E., Nelson, E. C., Duncan, A. E., Waldron, M., ... Heath, A. C. (2010). Posttraumatic stress disorder and alcohol dependence in young women. The Journal of Studies on Alcohol and Drugs, 71(6), 810-818.

Schnurr, P. P., \& Spiro, A., III (1999). Combat exposure, posttraumatic stress disorder symptoms and health behaviors as predictors of self-reported physical health in older veterans. Journal of Nervous and Mental Disease, 187(6), 353-359.

Schnurr, P. P., Spiro, A., III, \& Paris, A. H. (2000). Physician-diagnosed medical disorders in relation to PTSD symptoms in older male military veterans. Health Psychology, 19(1), 91-97.

Schwartz, A. C., Bradley, R., Penza, K. M., Sexton, M., Jay, D., Haggard, P. J., ...Ressler, M. D. (2006). Pain medication use among patients with posttraumatic stress disorder. Psychosomatics, 47(2), 136-142.

Shalev, A., Bleich, A., \& Ursano, R. J. (1990). Posttraumatic stress disorder: Somatic comorbidity and effort tolerance. Psychosomatics, 31, 197 203.

Silva, S., \& Maia, A. (2008). Experiências adversas na infância e na idade adulta em sujeitos com obesidade mórbida: $\mathrm{O}$ que é que tem mais “peso" para a adaptação actual? In I. Leal, J. L. 
P. Ribeiro, I. Silva, \& S. Marques (Orgs.), Actas do $7^{\circ}$ Congresso Nacional de Psicologia da Saúde (pp. 667-670). Porto, Portugal: Universidade do Porto.

Smith, J. W. (2000). Addiction medicine and domestic violence. Journal of Substance Abuse Treatment, 19, 329-338.

Stewart, S. H. (1996). Alcohol abuse in individuals exposed to trauma: A critical review. Psychological Bulletin, 120(1), 83-112.

Svetlicky, V., Solomon, Z., Benbenishty, R., Levi, O., \& Lubin, G. (2010). Combat exposure, posttraumatic stress symptoms and risk-taking behavior in veterans of the Second Lebanon War. Israel Journal of Psychiatry and Related Science, 47(4), 276-283.

Vieweg, W. V., Julius, D. A., Fernandez, A., Tassone, D. M., Narla, S. N., \& Paudurangi, A. K. (2006). Posttraumatic stress disorder in male military veterans with comorbid overweight and obesity: Psychotropic, anti-hypertensive and metabolic medications. Primary Care Companion. Journal of Clinical Psychiatry, 8, 25-31.

Walker, E. A., Gelfand, A., Katon, W. J., Koss, M. P., Korff, M. V., Bernstein, D., ...Russo, J. (1999). Adult health status of women with histories of childhood abuse and neglect. American Journal of Medicine, 107, 332-339.

Weg, M. W. V. (2011). Adverse childhood experiences and cigarette smoking: The 2009 Arkansas and Louisiana Behavioral Risk Factor Surveillance Systems. Nicotine \& Tobacco Research, 13(7), 616-622.
Westerink, J., \& Giarratano, L. (1999). The impact of posttraumatic stress disorder on partners and children of Australian Vietnam veterans. Australian and New Zealand Journal of Psychiatry, $33,841-847$.

Whitmire, L., \& Harlow, L. L. (1996). Childhood sexual abuse linked with adult substance use, victimization, and AIDS-risk. AIDS Education and Prevention, 8, 44-57.

Williamson, D. F., Thompson, T. J., Anda, R. F., \& Felitti, V. (2002). Body weight and obesity in adults and self-reported abuse in childhood. International Journal of Obesity and Related Metabolic Disorders, 26, 1075-1082.

Zen, A. L., Zhao, S., Whooley, M. A., \& Cohen, B. E. (2012). Post-traumatic stress disorder is associated with poor health behaviors: Findings from the heart and soul study. Health Psychology, 31(2), 194-201.

Zierler, S., Feingold, L., Laufer, D., Velentgas, P., Kantrowitz-Gordon, I., \& Mayer, K. (1991). Adult survivors of childhood sexual abuse and subsequent risk to HIV infection. American Journal of Public Health, 81(5), 572-575.
Recebido: 28/10/2011

$1^{a}$ revisão: 07/11/2012

Aceite final: 21/11/2012 